Cinémas

Revue d'études cinématographiques

Journal of Film Studies

\title{
Le petit glossaire « cinématographique » de la science cognitive
}

\section{Bernard Perron}

Volume 11, numéro 2-3, printemps 2001

Eisenstein dans le texte

URI : https://id.erudit.org/iderudit/024857ar

DOI : https://doi.org/10.7202/024857ar

Aller au sommaire du numéro

Éditeur(s)

Cinémas

ISSN

1181-6945 (imprimé)

1705-6500 (numérique)

Découvrir la revue

Citer cet article

Perron, B. (2001). Le petit glossaire « cinématographique » de la science cognitive. Cinémas, 11(2-3), 275-290. https://doi.org/10.7202/024857ar

\section{Résumé de l'article}

Il sera ici question du possible emprunt par les travaux de chercheurs en sciences cognitives de termes cinématographiques tels que "script ", " scénario ", " scène ", " cadre » ou " frame ». En s'intéressant aux images utilisées afin d'exprimer les mécanismes de l'esprit, à l'étymologie des mots ainsi qu'à la dimension narrative de la pensée, il s'agira de préciser les liens que peut cultiver la " science de l'esprit » avec le cinéma, cet « art de l'esprit ». 


\section{Le petit glossaire "cinématographique " de la science cognitive ${ }^{1}$}

\section{Bernard Perron}

\section{RÉSUMÉ}

Il sera ici question du possible emprunt par les travaux de chercheurs en sciences cognitives de termes cinématographiques tels que "script", "scénario ", "scène", "cadre" ou "frame". En s'intéressant aux images utilisées afin d'exprimer les mécanismes de l'esprit, à l'étymologie des mots ainsi qu'à la dimension narrative de la pensée, il s'agira de préciser les liens que peut cultiver la "science de l'esprit " avec le cinéma, cet " art de l'esprit ".

\section{ABSTRACT}

This article proposes to examine the possible borrowing of cinematographic terms like "script," "scenario," "scene," and "frame," in the research work of cognitive scientists. While focusing on certain images used to express the mechanics of the mind, the etymology of key-words, as well as the narrative dimensions of thinking, it attempts to clarify the relationship that the "science of the mind" might cultivate with the cinema, that "art of the mind."

Il sera essentiellement question ici de "scénario", de "script", de «scène", de «cadre " et de "frame». Ce qui se présente d'emblée comme un article sur le cinéma - je suis sûr que ces termes renvoient pour la majorité d'entre vous à ce dernier - consistera plutôt à un questionnement sur l'impact de cet art, de ce média sur la pensée et sur la production intellectuelle. Ma 
réflexion tire son origine d'une observation fort pertinente de Gilles Thérien :

Il est intéressant de noter que toutes les catégories de script, cadre, frame, soumises dans les travaux des chercheurs en science cognitive sont en fait empruntées au cinéma. L'histoire des idées devra un jour examiner le rapport entre ces deux événements (1992, p. 117).

Bien qu'il soit tentant de corroborer aussitôt ce rapport ${ }^{2}$, il est tout aussi nécessaire d'en souligner le travers. Car en bon sémiologue s'intéressant aux sciences cognitives, Thérien doit savoir qu'il y a toujours une pensée interprétante derrière la lecture d'un signe (ou d'un representamen) et que de la sorte, un même signe pourra avoir différents interprétants. Il ne s'agit donc pas tant de confirmer ledit rapport que d'étudier les éléments qui permettent de le noter. Pour ce faire, je m'intéresserai aux images utilisées afin d'exprimer les mécanismes de l'esprit, à l'étymologie des mots et à la dimension narrative de la pensée.

Il y a d'abord lieu de souligner que la relation entre les catégories du cinéma et celles de la science cognitive est aujourd'hui, et de façon usuelle, signalée. On a quà consulter le Larousse. Dictionnaire du français pour apprendre que le mot «scénario" définit en premier lieu la "rédaction des divers épisodes d'un film, sans aucune indication technique", et ensuite le "déroulement programmé d'une action" (1987, p. 936). Même chose dans Le Nouveau Petit Robert où le mot «script" renvoie d'abord au "scénario d'un film " et ensuite à la «séquence d'événements utilisée en intelligence artificielle pour représenter les connaissances" (1995, p. 2055). Cependant, cette relation est loin d'être attestée par les chercheurs en science cognitive et en intelligence artificielle.

Dans "A Framework for Representing Knowledge", article fondateur publié en 1975, Marvin Minsky introduit le concept de "frame" (ou de cadre) en le définissant comme "a datastructure for representing a stereotype situation" (1975, p. 212). Il débute son explicitation par l'étude de la vision. Il constate notamment que lorsqu'on entre dans une chambre, on peut sai- 
sir toute la scène d'un coup d'œil grâce à notre cadre-chambre (room-frame). Il intègre à son propos le concept de "scénario" lorsqu'il devient nécessaire de considérer des séquences d'actions. Comme cadre thématique (thematic frame), le scénariofête d'enfant (party scenario) permet par exemple de supposer une série typique d'activités allant de l'achat d'un cadeau à la dégustation du gâteau. Malgré les analogies possibles, Minsky élabore sa théorie sans notifier son présumé emprunt au glossaire cinématographique et, plus important encore, sans jamais justifier le choix de sa terminologie. C'est aussi le cas dans un ouvrage tout aussi fondateur de 1977, Scripts, Plans, Goals and Understanding de Foger C. Schank et Robert P. Abelson - ouvrage auquel fait référence Gilles Thérien dans une note qui accompagne son observation. Ces derniers inventent $\left[s i c^{3}\right]$ la notion de "script". "A script is a structure that describes appropriate sequences of events in a particular context " (Schank et Abelson, 1977, p. 41). Cette structure est divisée en scènes et en actions, actions que Jean Matter Mandler appellera plus tard des "scènes" de script" (1984, p. 77). Schank et Abelson conviennent que cette notion demeure semblable au «frame» de Minsky et je cite, "except that it is specialized to deal with event sequences" $\left(1975^{4}\right.$, cité dans Tannen, 1979 , p. 140). Il est tout de même curieux de découvrir que leur notion procède du cadre et non pas du scénario de Minsky ou, pour étayer l'hypothèse de départ, du script/scénario cinématographique. Dans cette perspective de traduction, on ne s'étonnera pas non plus que dans le texte français de Lector in Fabula, on traduise la "sceneggiatura" qu'utilise Umberto Eco (1979, p. $79^{5}$ ) pour renvoyer au "frame" de Minsky par le mot "scénario" (Eco, [1979], 1985, p. 99) et que dans L'Homme cognitif, Pierre Lecoq se serve de ce même mot pour traduire cette fois-ci le "script" de Schank et Abelson (Weil-Barais, 1993, p. 393). D'autre part, la dimension spatiale du «frame» de Minsky sera, elle, caractérisée par le concept de "scène" par Jean M. Mandler $\left(1984^{\circ}\right)$.

Il faut regarder du côté de l'anthropologie et remonter à la source pour trouver un premier lien explicite entre les catégories qui nous intéressent. C'est à Gregory Bateson, dans "A Theory of Play and Fantasy" publié en 1955, qu'on attribue la paternité 
du concept de "frame» ou plus spécifiquement de "psychological frame " (cadre psychologique). Bateson l'utilise pour expliquer comment les animaux et les humains sont capables de s'entendre sur le degré d'abstraction de leur message ou de leur action et comment, par exemple, ils sont capables d'échanger des signaux véhiculant le message: "ceci est un jeu ${ }^{8}$ ". Ici, notamment, les signaux de mon texte véhicule que «ceci est un raisonnement ". L'auteur de Steps to an Ecology of Mind assume qu'un cadre psychologique possède un certain degré de réalité et constate que: "In many instances, the frame is consciously recognized and even represented in vocabulary ("play," "movie," "interview," “job," "language," etc.) " (Bateson, [1955], 1972, p. 186-187). Et il se sert d'Hollywood afin d'exemplifier les paradoxes du cadre (c'est-à-dire au sujet du trompe-l'œil ", de la terreur et du fantasme ${ }^{10}$ ). Mais pour mieux définir son concept psychologique, Bateson utilise deux sortes d'analogies, dont "the physical analogy of the picture frame" (Bateson, [1955], 1972, p. 186). Entre autres, comme le cadre psychologique, le cadre d'un tableau ou d'une image est inclusif puisqu'il focalise la perception du spectateur (viewer). Il sert de prémisses puisqu'il indique à ce dernier qu'il ne doit pas user du même type de pensée, pour interpréter le tableau, que pour interpréter le papier peint hors du cadre. En tant que cadre extérieur, il délimite aussi un type logique puisqu'il circonscrit un fond sur lequel les figures doivent être perçues (suivant la psychologie de la gestalt). Puisque le concept qu'il essaie de définir n'est ni physique ni logique, Bateson admet toutefois que cette analogie est trop concrète.

\footnotetext{
Rather, the actual physical frame is, we believe, added by human beings to physical pictures because these human beings operate more easily in a universe in which some of their psychological characteristics are externalized. It is these characteristics which we are trying to discuss, using the externalization as an illustrative device ([1955], 1972, p. 187).
}

En définitive, ce n'est pas le fait que Bateson mentionne Hollywood et le film ${ }^{11}$ dans son article qui est important, mais précisément le moyen d'illustration (illustrative device). C'est en te- 
nant compte de cette extériorisation des caractéristiques psychologiques que l'emprunt au glossaire cinématographique des chercheurs en science cognitive semble s'expliquer.

La naissance du septième art, on le sait, tient au développement d'une technologie émanant du désir d'effectuer une recréation intégrale de la réalité, de produire une illusion parfaite du monde extérieur. Bazin appelait ce projet "le mythe du cinéma total" (1985, p. 19-24). À l'instar de la perspective artificielle en peinture et de la photographie, le cinéma changea la manière de percevoir le monde et d'en garder la trace. John L. Fell remarque dans Film and the Narrative Tradition qu'au sein de l'opinion populaire, la photographie est devenue dès le début $\mathrm{du} \mathrm{XX}^{\mathrm{e}}$ siècle un paradigme courant pour traduire les processus perceptifs et mentaux (Fell, 1974, p. 165). Il note que les nouveaux médias photographiques figurent, avec persistance, les idées que les écrivains se font de l'opération de l'esprit. Il renvoie à la mémoire chez Proust, "memory as accessible in the way a photographer "goes deliberately into his darkroom to develop the latent images in leisurely comtemplation" "(Fell, 1974 , p. 81). Dans cette foulée, les narratologues littéraires ont à leur tour largement invoqué l'appareil de prise de vues/son et les techniques cinématographiques. Dès 1948, dans son célèbre livre L'Âge du roman américain, Claude-Edmonde Magny compare et rapproche le style romanesque des écrivains français et américains de la fin du XIX ${ }^{\mathrm{c}}$ siècle et du début $\mathrm{XX}^{\mathrm{e}}$ avec le langage du septième art:

Le roman contemporain fait souvent usage de la mobilité de la caméra, autrement que pour découper le récit en plans différents. Comme le cinéma, il emploie volontiers travelling, fondu enchaîné et crossing-up [ou montage alterné] (Magny, 1948, p. 100).

\section{Ou encore :}

Nous allons voir que, pas plus que l'ellipse ou la technique objective, la succession de plans n'est un procédé nécessairement et effectivement limité au film, et que dans le roman contemporain (singulièrement chez les Américains, qui semblent en avoir fait un usage à peu 
près systématique) comme au cinéma, on peut parler de découpage (p. 80-81).

Jaap Lintvelt (Essai de typologie narrative: le point de vue) utilise toujours en 1980 la métaphore de «l'enregistrement pur et simple du monde extérieur» pour illustrer que «l'action romanesque n'est plus perçue par des acteurs, mais, pour ainsi dire, focalisée par une caméra" (cité dans Jost, 1987, p. 254). Ainsi, depuis que l'œil a vu le monde (re)présenté à travers le viseur d'une caméra, la perception a également trouvé son cadre ou son frame de référence. Et puisque cette dernière ne va pas sans une part de cognition, il n'y a qu'un pas entre le champ de vision et le champ de la connaissance ${ }^{12}$. De toute évidence, c'est à Hugo Münsterberg que l'on doit la corrélation la plus explicite entre le cinéma et la psychologie:

If this is the outcome of esthetic analysis on the one side, of psychological research on the other, we need only to combine the result of both into a unified principle: the photoplay tells us the human story by overcoming the forms of the outer world, namely, space, time, and causality, and by adjusting the events to the forms of the inner world, namely, attention, memory, imagination, and emotion ([1916], 1970, p. 74).

Dès 1916, le cinéma est présenté comme un "art de l'esprit». L'analogie marquée et accentuée dans le domaine artistique ne pouvait que trouver écho dans celui de la "science de l'esprit". Née en $1956^{13}$, l'intelligence artificielle (I.A.) fut elle aussi précédée par un désir, celui de récréer l'ensemble des facultés cognitives de l'homme. On pourrait dans ce cas-ci parler du «mythe de l'intelligence artificielle totale». Lorsque, à partir des toutes premières simulations de certaines fonctions mentales à l'aide de logiciels spécialisés ou de systèmes experts ${ }^{14}$, les chercheurs étudiant le fonctionnement de l'intellect voulurent développer un vocabulaire pour décrire les phénomènes qu'ils décelaient, ils trouvèrent des termes concrets déjà associés littéralement ou métaphoriquement aux processus de l'esprit. Cette explication n'est pas qu'hypothétique. Dans un courriel personnel en réponse à mes présentes interrogations, Marvin Minsky affirme que c'est plutôt le 
"picture-frame for holding a picture" qui lui a inspiré son concept de cadre - à l'instar de Bateson donc. Il écrit ensuite:

However, that certainly blurs into the cinematic frame notion. (When I was a small child, we had a $16 \mathrm{~mm}$ roll of Felix the Cat, and I spent some time drawing my own cartoons with a fine pen, on the transparent leader of that reel.) Certainly, I long had the idea of a "script" - but I think I usually said "plan" until reading Schank's work. Of course "script" (I think) was long used for plays before cinema came on the scene. [...] For scenario, you may be right. I needed some word for idea of a high-level, abstract sequence of situations before the details are filled in. I still can't think of a better word; "plot" almost works, but it feels at too high a level (Minsky, courriel personnel, 2 mars 1999).

Un détour par l'étymologie des mots en question éclaire davantage le commentaire de Minsky.

Dérivé du latin cadrus, "carrée, le mot "cadre» exprime à la fois l'idée d'une délimitation physique et d'une détermination abstraite. Tel que l'a montré avec nuance Bateson, le cadre peut être vu comme une structure qui soutient une image et comme un ensemble de prémisses qui encadre une interprétation. Selon le Dictionnaire historique de la langue française, le seul emploi courant du transitif du verbe "cadrer " demeure depuis 1912 celui de la photographie et du cinéma (1992, p. 320). Cependant, puisque les premiers chercheurs en science cognitive sont de langue anglaise, il est à la fois logique et plus pertinent de noter que dans le vocabulaire cinématographique anglais (contrairement à celui de la peinture et même de la photographie ${ }^{15}$ ), le "frame" d'une image est venu à désigner l'image elle-même ${ }^{16}$. On parle de "frames per second" et non de "pictures" ou de "photographs". The Oxford English Dictionary fait remonter cet emploi au milieu des années dix:

1916 E.W. Sargent Technique Photoplay (ed. 3) 362, Frame. A single photograph in the roll of film. A picture one inch wide by three-fourths on an inch high. There are sixteen of these frames to the foot (1989, p. 141). 
On peut toutefois supposer que des questions de nomenclature du support et du dispositif ou que des spécifications de brevets d'invention (pour Edison par exemple) ont dû jouer beaucoup plus tôt un rôle dans cette modification de sens ${ }^{17}$. Il n'en demeure pas moins que ce passage du cadre à l'image elle-même se rapproche encore plus de la notion cognitive de "frame" telle que l'a définie Minsky, c'est-à-dire comme un coup d'œil ou une appréhension globale d'une réalité complexe. Notre champ de vision étant très large et flou en périphérie, nous n'avons pas conscience de percevoir le monde à travers un cadre - ce que l'art nous rappelle continuellement. D'autre part, The Barnhart Dictionary of Etymology établit la filiation de l'expression "frame of mind " (état d'esprit) à Sir Richard Steele dans The Spectator de 1711. Périodique quotidien, The Spectator jetait un regard critique sur la société par l'entremise de spectateurs fictifs qui exaltaient les propres idées de Steele et des différents auteurs. À cause de son cadre fictionnel, The Spectator aurait annoncé l'ascension du roman anglais du dix-huitième siècle (The New Encyclopaedia Britannica, 1992, p. 78). Nous voilà reconduit aux propos de John L. Fell et de Claude-Edmonde Magny.

Le mot "scénario" est issu du latin scaenarium qui veut dire scène de théâtre. Il tient davantage son origine de l'italien scenario qui veut dire décor. Il est d'abord employé au théâtre et dans la littérature. Qu'on fasse ensuite remonter cet emploi à Feuillade en 1911 (Le Petit Robert, 1985, p. 648 ) ou à Méliès en 1907 (Giraud, 1958, p. 178) ou en 1902 (Raynauld, 1990), le terme français ou anglais est associé presque exclusivement au cinéma plusieurs décennies avant que Minsky l'adopte pour définir son "thematical frame». The Barnhart Dictionary of Etymo$\log y$ nous éclaire de nouveau. Il y est indiqué que: "The figurative sense of an outline of an imagined situation or chain of events is first recorded in Herman Kahn's Thinking about the Unthinkable (1962)"(1988, p. 966). Cet ouvrage nous plonge en pleine guerre froide. La première fois que le mot "scénario" y est employé, il l'est entre guillemets comme si Khan, un physicien nucléaire, notifiait son utilisation hors contexte (Khan, 1962, p. 133). Le scénario résulte là d'un "attempt to describe in more or less detail some hypothetical sequence of events" 
(1962, p. 150). C'est une intrigue fictionnelle (fictionnal plot) se voulant " one of many devices useful in stimulating and disciplining the imagination" (1962, p. 152). L'un des deux exemples de Khan, la guerre accidentelle, débute par une supposée crise. Un missile nucléaire soviétique est accidentellement mis à feu et touche l'Europe de l'Ouest. Croyant en une déclaration de guerre et sans vérifier les raisons de l'attaque, les États-Unis pourraient riposter immédiatement. Ce serait le début de la Troisième Guerre mondiale. Des dizaines de millions de gens seraient tués. Cependant, détail crucial malgré l'arme nucléaire, il n'y aurait pas d'Armageddon. Au lendemain de la guerre, le président des États-Unis enverrait une copie du livre World Peace Through World Law ${ }^{18}$ au président de l'Union soviétique en lui demandant de signer comme lui cet ouvrage, ce que ce dernier accepterait. Le reste du monde acquiescerait aussi à ce traité et la paix régnerait une fois de plus. Cette intrigue permet peut-être selon Khan d'envisager de meilleurs systèmes de contrôle afin d'éviter une guerre accidentelle, mais c'est aussi un scénario digne d'un "blockbuster" hollywoodien. On retrouve là la justesse du terme cinématographique. En général, le scénario précède et structure le film. C'est un texte virtuel. Il se présente, comme l'indique Jean-Paul Torök dans un essai sur le scénario, «comme le modèle, la simulation, la maquette pourrait-on dire, du film tel qu'il sera réalisé » (Torök, 1986, p. $202^{19}$ ).

Du latin scriptum et emprunté à la langue anglaise au début du XX ${ }^{e}$ siècle, "script" signifie écrit, type particulier de caractères typographiques. C'est pourquoi il définit toujours un document écrit. L'expression "to write a script" est datée de 1935 par The Barnhart Dictionary of Etymology (1988, p. 973). C'est tout à fait ce que font à la base Schank et Abelson. Ils écrivent des scripts pour SAM (Script Applier Mechanism), leur programme d'interprétation du langage naturel mis au point à l'époque à l'Université Yale. En plus d'élaborer l'ordre des scènes et des séquences d'actions, ces scripts tiennent compte d'un autre principe narratif: "A script must be written from a particular role's point of view" (Schank et Abelson, 1977, p. 42). On néglige souvent d'indiquer que leur fameux "script restaurant" est écrit à partir du point de vue d'un client. Qu'il s'agisse d'une 
connaissance schématique ou du texte d'une scène cinématographique à réaliser, le script sert de jalons à l'action. Du reste, le mot (anglais) "script" est attesté depuis 1897 comme le terme désignant le manuscrit de théâtre et depuis 1931 comme celui dactylographié d'un film (The Oxford English Dictionary, 1989, p. 741). Mais voilà, tel que me le fait savoir Robert P. Abelson, son emploi en science cognitive vient de la première et non de la seconde source:

I borrowed the term "script" with the theater in mind. I have had some acting experience, and it seemed apt, since much social interaction is constrained by the script of that situation (courriel personnel, 12 mars 1999).

Après avoir précisé qu'Abelson utilisait le terme avant qu'ils ne travaillent ensemble, Robert C. Schank atteste à son tour :

The reason I liked the word script was because of plays not movies; I always thought of scripts that we read in life to be just like scripts one memorizes in the theatre, reading ones role; why theater and not movies? Probably because I acted in plays as a child and since I grew up in NYC I attended the theater a great deal (courriel personnel, 10 mars 1999).

Les propos de Schank et Abelson occasionnent un important retour de la pensée. Tout raisonnement procède des connaissances, des expériences et des "cadres psychologiques" de chacun. Une hypothèse sera formulée et souvent examinée à partir de ses propres prémisses. Ainsi, et c'est exactement ce qui se produit avec l'observation initiale de Gilles Thérien, il est possible d'avoir en grande partie tort d'avoir raison. Car il faut bien avouer que pour un chercheur en cinéma, ledit emprunt au glossaire cinématographique va de soi, spécialement lorsqu'aucune information ne témoigne du contraire. On ne tombe pas toujours sur des passages comme celui-ci tiré de «Scenes-andframes semantics" de Charles J. Fillmore, que j'abrège:

The word scene that I have been using $[\ldots]$ is by no means to be associated solely with the prototype meaning of that word. Some of the things I would like to 
call scene are like that $[\ldots]$ Others are closer to a cinematic sense of scene [...] Other scenes [...] correspond [...] to the stage-direction sense of scene [...] Others scenes [are] historical [...] Other scenes involve a understanding of conditions [...] Other scenes have to do with body image [...] A still different kind of scene [...] is something we might refer to as an interactional scene (Fillmore, 1977, p. 72-74).

Difficile alors de rester cantonné dans son unique champ de recherche.

Enfin, je crois qu'il existe une dernière explication qui permet d'expliciter les raisons ayant pu inciter Thérien à présumer l'emprunt des chercheurs en science cognitive. À l'opposé des images mentales floues et incertaines que l'on conserve dans ses souvenirs, et contrairement à l'empreinte fixe de la photographie, le film garde une trace dynamique des images prélevées sur le réel. Cette dimension est fondamentale étant donné que les «frame», "cadre", "scène ", "script" et "scénario" ne peuvent pas être définis comme des structures statiques (voir Van Dijk, 1980, p. 236). Car malgré la diversité de la terminologie, chacune des notions introduites repose en fait sur la même théorie sousjacente: il s'agit de comprendre les caractéristiques typiques d'un événement ou d'un lieu à partir de la manière dont on saisit une sorte de récit. SAM (Script Applier Mechanism), le programme de Schank et Abelson, est un programme capable de comprendre des histoires simples et standards (par exemple le script-restaurant). Cette faculté artificielle de comprendre et de connaître n'est certes pas étrangère à l'intelligence humaine, intelligence pour qui «la forme caractéristique de l'expérience construite (et de la mémoire que j'en ai) est la forme narrative" (Bruner, 1991, p. 69). Sur cet élan, on voit poindre le septième art. En plus de la fameuse narrativité qu'il a bien chevillée au corps (dixit Metz), les images et les sons que le cinéma propose s'inscrivent illico dans notre disposition innée au récit. Quand je repense au passage d'un roman que j'ai lu, je ne me souviens généralement pas des mots mais des images que je m'étais formées lors de ma lecture. C'est un peu la même chose qui se produit pour les souvenirs de ses propres actions passées et de celles que 
j'entreprends. Les souvenirs que je me remémore se présentent comme des séquences de film. D'ailleurs, l'expression populaire ne dit-elle pas qu'on se souvient du «film des événements». De la sorte, beaucoup plus que les signes conventionnés, les signes analogiques que l'on retrouve dans le film se rapprochent de la réalité quotidienne et de notre propension narrative protolinguistique. C'est pourquoi la conceptualisation du cinéma a pu tenir une place de choix au sein de la théorie en science cognitive. Ici, j'anticipe une contradiction: certains modèles étudiant la représentation de la connaissance en mémoire sémantique supposent que les textes, faits et événements sont emmagasinés sous forme de propositions et non sous formes d'images (voir Fortin et Rousseau, 1993, p. 391-393 ).C'est une manière de voir l'abstraction de la signification. En revanche, c'est aussi négliger notre capacité à conserver et à former ${ }^{20}$ intuitivement des images.

Bien au-delà des catégories que je viens de considérer et du raisonnement que je viens d'effectuer, la science cognitive, l'intelligence artificielle et le cinéma cultivent d'excellentes relations. Leurs points de convergence sont de plus en plus nombreux avec la réalité virtuelle. Dans "The Future Merging of Science, Art, and Psychology ", Minsky fait même remonter cette nouvelle technologie aux débuts du cinéma d'animation ([1990], 1993). La science et l'art partagent effectivement un noyau identique: l'esprit, c'est-à-dire le site premier de l'intermédialité. Une conclusion, à n'en pas douter, peu originale mais qu'il ne faut définitivement pas négliger.

Université de Montréal

\section{NOTES}

1. Cet article a été rédigé avec le support d'une bourse postdoctorale du Conseil de recherches en sciences humaines du Canada. L'auteur tient à remercier Marvin Minsky, Robert P. Abelson et Robert C. Schank, ainsi que Jean Châteauvert, Pierre Fontaine et Sébastien Ruffo pour leur contribution.

2. D'ailleurs, la tentation est si forte que c'est justement ce que j'ai fait dans un passage de ma thèse (La Spectature prise au jeu. La narration, la cognition et le jeu dans le cinéma narratif, thèse de doctorat, Université de Montréal, 1997, p. 94-99) et lors de ma communication présentée au premier colloque international du Centre de recherche sur l'intermédialité (CRI) de l'Université de Montréal. 
3. "Scripts, although invented to handle a different but related problem, from the basis of the answer to the representation of certain complex nouns as well " (Schank et Abelson, Scripts, Plans, Goals and Understanding, Hillsdale, N.J. : Lawrence Erlbaum Associates, 1977, p. 16).

4. Il s'agit d'un article qui précède leur ouvrage.

5. Je dois cette précision à un évaluateur externe.

6. Mandler distingue par des guillemets la notion de "scène" de celle de "scène", qui renvoie, on vient de le voir, à l'action.

7. " $[\mathrm{I}] \mathrm{t}$ is (or delimits) a class or set of messages (or meaningful actions)" (Bateson, "A Theory of Play and Fantasy", Steps to An Ecology of Mind, New York: Ballantine Books, [1955] 1972, p. 186).

8. Précisons que ma récapitulation de l'article repose sur la traduction française du texte publiée en 1977 (p. 211). Je préfere toutefois me référer à la version originale anglaise pour les questions de citation.

9. "Hollywood film -makers spend millions of dollars to increase the realism of shadow" (Bateson, "A Theory of Play and Fantasy", Steps to An Ecology of Mind, New York: Ballantine Books, [1955] 1972, p. 182)

10. "A man experiences the full intensity of subjective terror when a spear is flung at him out of the 3D screen or when he falls headlong from some peak created in his own mind in the intensity of nightmare. At the moment of terror there was no question of "reality," but still there was no spear in the movie house and no cliff in the bedroom. The images did not denote that which they seem to denote, but theses images did really evoke that terror which would have been evoked by a real spear or a real precipice. By a similar trick of self-contradiction, the film-makers of Hollywood are free to offer to a puratinical public a vast range of pseudosexual fantasy which otherwise would not be tolerated" (Bateson, "A Theory of Play and Fantasy", Steps to An Ecology of Mind, New York: Ballantine Books, [1955] 1972, p. 183).

11. Umberto Eco se réfère aussi directement au cinéma lorsqu'il explique les tenants du "scénario intertextuel" (Lector in fabula: le rôle du lecteur, Paris: Éditions Grasset et Fasquelle, [1979] 1985, p. 105).

12. Montrer, c'est d'abord donner à voir. Par contre, c'est également donner à savoir. Cela constitue assurément l'une des observations les plus pertinentes de l'application du concept genettien de focalisation au cinéma. L'interdépendance entre l'optique et la connaissance est également une thèse développée en théories des arts visuels. On pense aussi à la corrélation entre la machine militaire et la machine de vision chez Paul Virilio (Guerre et cinéma I. Logistique de la perception, Paris : Cahiers du cinéma/Éditions de l'Étoile, 1984).

13. Le cognitivisme serait né le 11 septembre 1956, jour de la présentation d'une communication de Noam Chomsky: "Three models of language " (Small, "Introduction: Cognitivism and Film Theory", Journal of Dramatic Theory and Criticism, vol. 6, $\mathrm{n}^{\circ}$ 2, printemps, 1992 : copie électronique sans pagination). L'intelligence artificielle aurait, elle, aussi vu le jour en 1956 alors que deux chercheurs, A. Newell et $\mathrm{H}$. A. Simon, présentaient lors d'un congrès le premier programme effectif qui simulait la démonstration d'un théorème (Weil-Barais (direction), L'Homme cognitif, Paris: Presses universitaires de France, 1993, p. 49).

14. Des « logiciels travaillant sur une base de connaissance et capable, par des traitements logiques de nature heuristique, de simuler les raisonnements et les prises de décision d'un expert humain " (Le Nouveau Petit Robert, Paris: Dictionnaires Le Robert, 1985). Voir aussi Weil-Barais (direction), L'Homme cognitif, Paris: Presses universitaires de France, 1993, p. 46-52. 
15. Selon The Focal Encyclopedia of Photography: "Frame. Single image in a series on a strip of film. Applied mainly to cinematography (for example in defining film size and camera speed), but also used in still photography where for instance a single exposure on a $35 \mathrm{~mm}$. film is often referred as a frame" (New York: Focal Press, 1965, p. 677).

16. Je dois cette observation à Marvin Minsky (courriel personnel, 2 mars 1999).

17. Cette modification de sens demande à être explicitée. Je n'ai trouvé aucun ouvrage qui en révélait les tenants.

18. Livre de Granville Clark et Louis B. Sohn (Cambridge:Harvard University Press, 1958).

19. Je tire ce dernier passage de Torök du mémoire de maîtrise de Pierre Fontaine sur le théâtre expérimental québécois et le cinéma ("Spactator in fabula». Le théatre expérimental et l'univers du spectateur modèle entre 1980 et 1985. Mémoire de maîtrise en études françaises, Université de Montréal, 1999, p. 10). Fontaine y discute entre autres des scénarios communs et intertextuels d'Eco.

20. "Il ne s'agit évidemment pas de dire que nous avons des "images dans notre tête". Cependant, nous disposerions d'une capacité spécifique nous permettant de former des représentations analogues, c'est-à-dire qui garderaient généralement les propriétés des réalités physiques" (Fortin et Rousseau, Psychologie cognitive. Ine approche de traitement de l'information, Québec: Télé-Université, 1993, p. 299).

\section{OUVRAGES CITÉS}

Abelson, Robert P. "Psychological Status of the Script Concept" [1975] dans American Psychologist, vol. 36, n' 7, July 1981, p. 715-729.

Barnhart Dictionary of Etymology (The). New York: The H.W. Wilson, 1988.

Bateson, Gregory. "A Theory of Play and Fantasy" dans Steps to an Ecology of Mind. New York: Ballantine Books, [1955] 1972, p. 177-193. En français: Vers une écologie de l'esprit. Paris: Seuil, 1977, p. 209-224.

Bruner, Jérôme ... Car la culture donne forme à l'esprit. De la révolution cognitive à la psychologie culturelle. Paris: Éditions Eshel, 1991.

Dictionnaire historique de la langue française. Paris: Dictionnaires Le Robert, 1992.

Eco, Umberto. Lector in fabula. La cooperazione interprativa nei testi narrativi. Milan: Bompiani, 1979.

Eco, Umberto. Lector in fabula : le rôle du lecteur. Paris : Éditions Grasset et Fasquelle, [1979], 1985.

Fell, John L. Film and the Narrative Tradition. Berkeley: University of California Press, 1974.

Fillmore, Charles J. "Scenes-and-Frames Semantics" dans Linguistic Structure Processing, A. Zampolli (direction), New York: North-Holland Publishing Company, 1977, p. $55-81$.

Fortin, Claudette et Robert Rousseau. Psychologie cognitive. Une approche de traitement de l'information. Québec: Télé-Université, 1993.

Giraud, Jean. Le lexique français du cinéma des origines à 1930. Paris: Centre National de la Recherche scientifique, 1958.

Jost, François. "Propositions pour une narratologie comparée" dans Mana, n" 7 , 1987, p. 251-265.

Khan, Herman. Thinking About the Unthinkable. New York: Avon Books, 1962. 
Larousse. Dictionnaire du français. Paris: Larousse, 1987.

Magny, Claude-Edmonde. L'Âge du roman américain. Paris: Seuil, 1948.

Mandler, Jean Matter. Stories, Scripts, and Scenes: Aspects of Schema Theory. Hillsdale, N.J. : Lawrence Erlbaum Associates, 1984.

Metz, Christian. Le Signifiant imaginaire. Paris: Christian Bourgois Éditeur, [1977] 1984.

Minsky, Marvin. "A Framework for Representing Knowledge" dans The Psychology of Computer Vision, P.H. Winston (eds). New York: McGraw-Hill Books, p. 211-280, 1975.

Minsky, Marvin. "The Future Merging Of Science, Art, and Psychology". Applied Artificial Intelligence, vol. 7, [1990] 1993.

Münsterberg, Hugo. The Film. A Psychological Study. The Silent Photoplay in 1916. New York: Dover Publications Inc, [1916] 1970.

Nouveau Petit Robert (Le). Paris: Dictionnaires Le Robert, 1995.

Oxford English Dictionary (The). Oxford: Clarendon Press, 2e édition, 1989.

Petit Robert (Le). Paris: Dictionnaires Le Robert, 1985.

Raynauld, Isabelle. Le Scénario comme texte. Histoire, théorie et lecture(s) du scénario, de Georges Méliès à Margerite Duras et Jean-Luc Godard. Thèse de doctorat, Université de la Sorbonne Nouvelle-Paris VII ,1990 (non publiée).

Schank, Roger C. et Abelson, Robert P. "Scripts, plans and Knowledge" dans Advanced Papers of the Forth International Joint Conference on Artificial Intelligence. Tibilis/ Cambridge, Mass. : Artificial Intelligence Lab, vol. 1, p. 151-157, 1975.

Schank, Roger C. et Robert P. Abelson. Scripts, Plans, Goals and Understanding. Hillsdale, N.J. : Lawrence Erlbaum Associates, 1977.

Tannen, Deborah. "What's in a Frame? Surface Evidence for Underlying Expectations" dans New Directions in Discourse Processing. Volume II: Advances in Discourse Processes, édité par R. O. Freedle (direction). Norwood: Ablex Publishing Corporation, 1979, p. 137-181.

Thérien, Gilles. "La lisibilité au cinéma" dans Cinémas (Cinéma et réception), vol. 2,

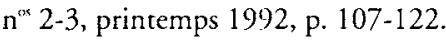

Van Dijk, Teun A. Macrostructures. Hillsdale, N.J. : Lawrence Erlbaum Associates, 1980.

Weil-Barais, Annick (direction). L'Homme cognitif. Paris: Presses universitaires de France, 1993.

\section{BIBLIOGRAPHIE COMPLÉMEN'TAIRE}

De Montvalon, Christine. Les mots du cinéma. Paris: Belin, 1987.

Encyclopedia of Photography. New York: Focal Press, 3e édition, 1993.

Focal Encyclopedia of Photography (The). New York: Focal Press, 1965.

Fontaine, Pierre. "Spectator in fabula". Le théâtre expérimental et l'univers de son spectateur modèle entre 1980 et 1985. Mémoire de maîtrise en études françaises, Université de Montréal, 1999 (non publié).

Nouveau dictionnaire étymologique et historique. Paris: Référence Larousse, 1971.

Pavis, Patrice. Dictionnaire du théâtre. Paris: Messidor/Éditions Sociales, 1987.

Perron, Bernard. La Spectature prise au jeu. La narration, la cognition et le jeu dans le cinéma narratif. Thèse de doctorat, Université de Montréal, 1997 (non publiée).

Le petit glossaire «cinématographique» de la science cognitive 
Small, Edward S. "Introduction: Cognitivism and Film Theory" dans Journal of Dramatic Theory and Criticism, vol 6, $\mathrm{n}^{\circ}$ 2, printemps 1992, p. 165-172.

Spottiswoode, Raymond. Film and its Techniques. Berkeley: University of California Press, $2^{\circ}$ édition, 1968.

Törok, Jean-Paul. Le scénario. L'art d'écrire un scénario. Bruxelles: Éditions de l'Université de Bruxelles, 1986.

Trésors de la langue française. Paris: Gallimard, Centre national de recherche scientifique, 1992.

Virilio, Paul. Guerre et cinéma I. Logistique de la perception. Paris: Cahiers du cinéma/Éditions de l'Étoile, 1984. 